\title{
Associations between serum biomarkers of cartilage metabolism and serum hyaluronic acid, with risk factors, pain categories, and disease severity in knee osteoarthritis: a pilot study
}

Christos Papaneophytou ${ }^{1}$, Ana Alabajos-Cea ${ }^{2,3}$, Enrique Viosca-Herrero ${ }^{3}$, Carme Calvis ${ }^{4}$, Marta Costa ${ }^{4}$, Andreas E. Christodoulides ${ }^{5}$, Alexander Kroushovski ${ }^{5,6}$, Alkis Lapithis ${ }^{5}$, Vaia Maligianni Lapithi ${ }^{5}$, loannis Papayiannis ${ }^{5}$, Andreas Christou ${ }^{5}$, Ramon Messeguer ${ }^{4}$, Christoforos Giannaki ${ }^{1 *}$ and Kyriacos Felekkis ${ }^{1 *}$

\begin{abstract}
Background: Specific serum biomarkers of cartilage metabolism such as cartilage oligomeric matrix protein (sCOMP) and procollagen type II C-terminal propeptide (SPIICP) as well as hyaluronan (SHA), a biomarker of synovitis, have been implicated in the pathophysiology of knee osteoarthritis $(\mathrm{OA})$. However, the associations of these biomarkers with the severity of the disease and OA risk factors, including age and obesity remain inconclusive. This analysis examines the associations between these serum biomarkers and the radiographic severity of OA and knee pain, as wells as obesity, the age and gender of the participants, and other OA risk factors.

Methods: From 44 patients with early knee OA and 130 patients with late knee OA we analyzed the radiographic severity of the disease using the Kellgren and Lawrence $(\mathrm{KL})$ grading system. Moreover, 38 overweight healthy individuals were used as a control group. Specific information was collected from all participants during their recruitment. The levels of the three serum biomarkers were quantified using commercially available ELISA kits. Serum biomarkers were analyzed for associations with the average $\mathrm{KL}$ scores and pain in both knees, as well as with specific OA risk factors.

Results: The levels of sCOMP were elevated in patients with severe late OA and knee pain and correlated weakly with OA severity. A weakly correlation of sHA levels and OA severity OA was observed. We demonstrated that only sPIICP levels were markedly decreased in patients with late knee OA suggesting the alterations of cartilage metabolism in this arthritic disease. Moreover, we found that SPIICP has the strongest correlation with obesity and the severity of OA, as well as with the knee pain at rest and during walking regardless of the severity of the disease. ROC analysis showed that the area under the ROC curve (AUC) was 0.980 (95\% Cl: 0.945-0.995; $p<0.0001$ ), suggesting high diagnostic accuracy of sPIICP. Interestingly, gender and age had also an effect on the levels of sPIICP.
\end{abstract}

*Correspondence: giannaki.c@unic.ac.cy; fellekis.k@unic.ac.cy

${ }^{1}$ Department of Life and Health Sciences, School of Sciences and Engineering, University of Nicosia, 2417 Nicosia, Cyprus

Full list of author information is available at the end of the article

(c) The Author(s) 2022. Open Access This article is licensed under a Creative Commons Attribution 4.0 International License, which permits use, sharing, adaptation, distribution and reproduction in any medium or format, as long as you give appropriate credit to the original author(s) and the source, provide a link to the Creative Commons licence, and indicate if changes were made. The images or other third party material in this article are included in the article's Creative Commons licence, unless indicated otherwise in a credit line to the material. If material is not included in the article's Creative Commons licence and your intended use is not permitted by statutory regulation or exceeds the permitted use, you will need to obtain permission directly from the copyright holder. To view a copy of this licence, visit http://creativecommons.org/licenses/by/4.0/. The Creative Commons Public Domain Dedication waiver (http://creativeco mmons.org/publicdomain/zero/1.0/) applies to the data made available in this article, unless otherwise stated in a credit line to the data. 
Conclusion: This study revealed the potential of serum PIICP to be used as a biomarker to monitor the progression of knee OA, however, further studies are warranted to elucidate its clinical implication.

Keywords: Knee osteoarthritis, Biomarkers, COMP, HA, PIICP, OA risk factors, obesity

\section{Introduction}

Osteoarthritis $(\mathrm{OA})$ is one of the main causes of pain and disability with knee OA being a leading cause of disability among older adults globally affecting the life quality of patients [1]. The disease is characterized by joint pain and progressive degeneration of articular cartilage involving remodeling of all joint tissues (i.e., bone, synovium, ligaments) and joint space narrowing (JSN) [2, 3]. Therefore, from a physio-pathological perspective, knee $\mathrm{OA}$ is a whole joint disease involving structural modifications of the articular cartilage [3] and subchondral bone [4]. Knee OA also involves the disruption of the synovial membrane, meniscus, and the infrapatellar fat pad (IFP) [5]. The estimated prevalence of OA among adults over 60 years of age or older is approximately $13 \%$ in women and $10 \%$ in men [6]. The major risk factors for developing $\mathrm{OA}$, in addition to age, are gender (female sex), injury, obesity, genetic factors, and high workload [7, 8]. Lifestyle factors including physical activity and exercise have been associated with knee OA, although the evidence is inconsistent [9].

At present, the treatment of OA mainly depends on knee replacement surgery and approaches to reduce symptoms and/or pain [10], while there are no therapies and/or medication approved by regulatory authorities that alter the onset or progression of OA structural damage. The currently available treatments have only moderate effects, and therefore, patients are not satisfied with their efficacy [11]. Due to the failure of the available medications to treat knee OA, the number of joint replacement surgeries is increasing by $\sim 10 \%$ annually [12]. The anatomical severity of OA is usually assessed by clinical evaluation of pain, joint stiffness, and limitations in physiological function as well as by joint imaging using standard radiographs (X-ray, and magnetic resonance imaging), using the Kellgren and Lawrence scale [13], which is known as the KL grading system (0-4). A KL grade of 0 indicates an intact joint without any features of OA, and the subsequent grades 1-4 represent increasing severity of the disease, and a score $>2$ is indicative of OA being present. However, radiography reveals only changes in bone and cartilage, which tend to occur late in the disease [14].

Another way to monitor structural changes in $\mathrm{OA}$ is by measuring molecular markers (biomarkers) that are released into the blood and other biological fluids (e.g., urine) during the turnover of tissue [15]. In the past few years, the potential of molecules involved in the bone and cartilage metabolism as biomarkers for knee OA has been investigated (for a review on the topic see [16]). These biomarkers can be determined using commercially available ELISA kits [17]. Biomarkers in osteoarthritis can be categorized using BIPED (Burden of Disease, Investigative, Prognostic, Efficacy of Intervention, and Diagnostic) classification scheme proposed by the Osteoarthritis Biomarkers Network [18] and could be used to detect and monitor bone and cartilage turnover, as well as synovial metabolism for the evaluation of the pathophysiological processes that lead to both joint failure and pain in OA patients [19].

Specific biomarkers have been related to the presence and/or severity of knee OA in cross-sectional studies; whereas longitudinal studies have revealed some markers that could predict OA progression [15]. In the cases of knee OA, a biomarker could be an operator (effector molecule) and/or the result of joint damage. For example, cartilage oligomeric matrix protein (COMP), an important degradation product of articular cartilage is associated with OA severity [20]. COMP is a pentameric non-collagenous glycoprotein primarily identified in cartilage $[8,9]$, which is a member of the family of thrombospondin and acts as a catalyst in collagen formation [21]. Furthermore, the association of the levels COMP, with the radiographic severity of knee OA has been reported [22]. Also, in the case of cartilage extracellular matrix fragments, hyaluronan (HA) that reflects the extent of synovitis [23], may serve as both a biomarker and stimuli for the innate immune chronic wound healing response in the OA joint [24]. $\mathrm{HA}$ is a non-sulfated glycosaminoglycan consisting of alternately repeating D-glucuronic acid and $\mathrm{N}$-acetylglucosamine units. HA is abundantly in articular cartilage and synovial fluid and it is partly responsible for lubrication and viscoelasticity of the synovial $[25,26]$. $\mathrm{HA}$ also regulates several processes in the articular cartilage, synovial fluid, and subchondral bone (reviewed in [27]). Interestingly, both the concentration and chain length of HA are reduced in synovial fluid in patients with knee OA [26] suggesting that the low viscosity of the synovial fluid may cause the wear-and-pain associated with the disease [28]. Previous studies have also demonstrated that HA level in the circulation is associated with the severity of OA (KL grade) [29], age of patients $[29,30]$. 
Other studies have focused on the synthesis and degradation of type II collagen to identify biochemical markers for OA [7]. Type II collagen is a major structural protein of the cartilage and in combination with other collagen types and non-collagenous proteins, including COMP, forms a tensile network for cartilage [7]. The potential of serum procollagen type II C-terminal propeptide (sPIICP), which is cleaved from cartilage type II procollagen following the release of newly synthesized procollagen into the matrix $[15,31]$ as a biomarker for monitoring the progression of OA has also been investigated [32]. While a large number of studies have been pursued to identify disease-specific biomarkers for OA, only a very limited number have been identified [24]. A successful biomarker should facilitate the evaluation of disease progression, be easily determined using commercially available assay kits, and may help patients to understand their condition [33].

This work aimed to determine the levels of two biomarkers of cartilage metabolism namely COMP, a biomarker of cartilage degradation, and PIICP, a biomarker of cartilage synthesis as well as hyaluronan, a biomarker of synovitis in serum samples from healthy individuals and patients with different degrees of severity of knee OA. As suggested by other studies, we hypothesized that the concentrations of these biomarkers are correlated with the severity of knee OA and might predict the progression of the disease. Thus, a major objective of this work was to examine the relationship between specific biomarkers in circulation and clinical diagnosis. Combining biochemical markers with other risk factors (i.e., obesity, occupational risk factors, age, gender, etc.), may facilitate better monitoring of the progression of the disease and help in identifying asymptomatic knee OA patients.

\section{Materials and methods}

\section{Study population and clinical assessment}

A total of 38 healthy individuals at high risk of developing OA, 44 patients with early knee OA (EOA), and 130 patients with late (established) knee OA (LOA) were recruited as a random population sample to evaluate the correlation of biomarker levels with the severity of knee OA.

\section{Healthy subjects and patients with early knee osteoarthritis}

A total of 82 individuals have been recruited at Hospital La Fe, Valencia, Spain, from Oct 2018 until Dec 2020. Participants were volunteers attending the hospital for other reasons different from knee issues, mainly for a routine medical checkup. They were informed about the project, and the subjects interested in participating were filtered and classified according to the inclusion / classification criteria (see below). A clinical evaluation consisting of physical examination, patient-based questionnaires, radiographs (X-ray and/or Magnetic Resonance Image-MRI), and collection of blood samples was performed on each subject. After this clinical evaluation, subjects were divided into patients with EOA and healthy subjects. EOA was defined according to the criteria proposed by Luyten [34] which are intended only for research purposes and they are the most precise criteria for the diagnosis of EOA described in the literature. It should be pointed out that the patients have not progressed to advanced OA or any other arthropathy during the implementation of this study.

The inclusion criteria for EOA patients were (i) age $\geq 40$ years, (ii) $K L$ grade $0-1$, weight-bearing (at least 2 projections: PA fixed flexion and skyline for patellofemoral OA), iii) patient-based questionnaires (see section 2.2 "Clinical assessment"), and (iv) patients should present joint line tenderness or crepitus in the clinical examination. The group of patients with early OA included 44 patients ( 31 women, 13 men). All patients in this group had chronic daily pain of the knee for at least 6 months.

For the healthy group, the inclusion criteria were (i) age $\geq 40$ years; (ii) body mass index (BMI) $\geq 25 \mathrm{~kg} / \mathrm{m}^{2}$; and (iii) $\mathrm{KL}$ grade 0 . Healthy subjects $(n=38)$ at high risk of developing knee OA included 23 women and 15 men. Obesity has been associated with the onset and development of OA. For this reason, we chose it as an easily identifiable risk factor for filtering healthy subjects at risk of developing knee OA. None of the healthy individuals had evidence of knee OA assessed by clinical examination, questionnaire, and X-ray and/or MRI films of both knees.

Exclusion criteria were the same for both groups: (i) any cognitive disability that hindered viewing of the audio-visual material; (ii) illiteracy; (iii) comprehension or communication difficulties, (iv) insufficient Spanish language comprehension to follow measurement instructions; (v) presence of any rheumatic, autoimmune or infectious pathology. Individuals with diseases that might increase the levels of sCOMP and/or sHA such as cardiovascular diseases [35] and inflammatory diseases [36] were excluded from this study.

\section{Patients with established (late) osteoarthritis}

A total of 130 patients with late (established) knee OA (LOA) (98 women, 32 men) undergoing knee replacement surgery at Apollonion Hospital, Nicosia, Cyprus, were enrolled in this study from July 2018 until Jan 2020. The minimum criteria for knee replacement therapy (KTR) were significant, prolonged symptoms including intractable pain affecting the quality of life of patients, with supporting clinical and radiological signs [37]. 
Recruitment of patients was carried out by clinicians and OA was defined according to the American College of Rheumatology criteria (https://www.rheumatology. org/) for the classification and reporting of osteoarthritis of the knee [38]. The inclusion criteria for this group were as follows: age $\geq 50$ years, knee pain, radiological evidence ( $x$-ray images) of OA, crepitus audible/ palpable, and stiffness lasting under $30 \mathrm{~min}$. Patients with post-traumatic osteoarthritis, arthritis due to any autoimmune, infective, or inflammatory rheumatological conditions were also excluded from the study. All women were postmenopausal, and all OA patients were without treatment that could interfere with bone metabolism [39]. All patients had chronic daily pain of the knee for at least 6 months.

\section{Clinical assessment}

Demographic and clinical data, including age, weight, height, and clinical symptoms of the knee joints, were recorded. BMI was calculated as weight $(\mathrm{kg})$ divided by height $(\mathrm{m})^{2}$. The Kellgren and Lawrence (K\&L) scoring system (0-4) was used to assess the radiographic severity of OA while in both centers the same radiologist, with more than 10 years of experience in musculoskeletal system radiology, examined all the radiographs, and described the KL for each subject $[13,40]$. Healthy individuals had a KL grade of 0 in both knees while the patients of the EOA group had a KL score of 0 or 1 . Moreover, in this study, patients with established OA were divided into a mild group (KL grade 2 in at least one knee), and a severe group (KL grade 3 or 4 in at least one knee). In this study, because the majority of patients with established OA had both knees affected, the mean KL grade of both knees was used in the analysis (discussed further in "Results").

All participants were also interviewed regarding pain in both knees by asking: "Have you experienced left or right knee pain in the past months/years, during walking and/or at rest?". Knee pain at rest and during walking (before surgery for the patients of the late OA group) was assessed using the visual analog scale (VAS), an 11-point $(0-10)$ numerical rating scale $(0=$ no pain; $10=$ worst pain) [41]. All answers were recorded specifically for each knee [answer possibilities were: (1) no; (2) yes, in the right knee; (3) yes, in the left knee; or (4) yes, in both knees]. Individuals were defined as that i) without pain if they indicated a VAS score of zero and ii) with pain if they indicated a score more than zero. For data analysis in this work the average pain in both knees was used and for the graphical depiction of associations with biomarkers knee pain was divided into tertiles resulting in three groups i.e., low (0.5-3.5), moderate (4.0-7.0), and severe (7.5-10).
Patients were asked to participate in this research project with a voluntary decision and they should be competent to understand what is involved and thus, all patients provided written informed consent before study enrolment. A questionnaire was also prepared to collect specific information from each patient during their recruitment while we got approval from the Cyprus National Bioethics Committee (EЕBK/ЕП/2018/19) and the Ethics Committee on Drug Research of the University and Polytechnic Hospital La Fe, to perform the study. Family history of OA was assessed, as suggested in the literature [42], by the question "Have any of your closest relatives (including children, siblings, parents, and grandparents) had OA?" Response options: "Yes, one individual", "Yes, two or more individuals", "No", or "I do not know". Both responses "Yes, one individual" and "Yes, two or more individuals" were categorized as having a family history of OA.

Occupational physical exposure (occupational OA risk) was evaluated using the question: "For the job or occupation you had for the longest time, did you do any of the following nearly every day?" with the following answer options: "bending for 2 hours or more", "walking for 2 hours or more over level ground", "kneeling for $30 \mathrm{~min}$ utes or more", "squatting for 30 minutes or more", "climbing a total of 5 or more flights of stairs", "lifting or moving objects of $10 \mathrm{~kg}$ or heavier", "driving a car for 4 hours or more", "none of the above". The occupational risk was included as a categorical variable (yes/no) in statistical analyses. This question has previously been used in [42] Participants reported if they had had an injury on their knees and/or hips that caused them to visit a doctor, and if they had had previous surgery on their knee(s) and/or hip(s). Participants with a previous knee injury that could not lead to OA, i.e., soft tissue injury without a fracture that could lead to arthritis or at least without a fracture involving the joint surfaces which could lead to arthritis were included in this study.

\section{Sample collection and determination of biomarkers levels}

For the collection of blood, separation of serum, and long storage of the samples we followed the rules proposed by the Standard Operating Procedures Internal Working Group (SOPIWG)/ Early Detection Research Network (EDRN) for specimen collection (including blood samples and management for biomarker discovery and validation) [43]. All samples were stored at $4{ }^{\circ} \mathrm{C}$ to prevent hemolysis and processed within $4 \mathrm{~h}$ after collection. The samples were maintained at $2-8^{\circ} \mathrm{C}$ while handling. Serum samples were stored in $0.5-1.0 \mathrm{~mL}$ aliquots, at $-80^{\circ} \mathrm{C}$. Also, freeze-thaw cycles of the samples were avoided. All samples used were clear and transparent. Serum samples from healthy individuals and patients with EOA obtained 
at Hospital La Fe (Valencia, Spain) were stored and processed by the Biobanco La Fe (PT17/0015/0043), following standard operating procedures with the appropriate approval of the Ethics and Scientific Committees.

Serum biomarker levels were determined in the following way: After thawing, serum samples were centrifuged at $2500 \mathrm{~g}$ for $10 \mathrm{~min}$ at $4{ }^{\circ} \mathrm{C}$ and then diluted 1:100 for COMP, 1:8 for HA, 1:4 for PIICP. Determination of COMP and HA levels was carried out using the Human COMP Quantikine ELISA Kit (assay range: $0.2-10 \mathrm{ng} /$ $\mathrm{mL}$ ) and Hyaluronan Quantikine ELISA Kit (assay range: $0.6-40 \mathrm{ng} / \mathrm{mL}$ ), respectively from R\&D systems (Minneapolis, USA). PIICP levels were determined using the Human Procollagen II C-Terminal ProPeptide (PIICP) CLIA Kit (assay range: $15.63-1000 \mathrm{pg} / \mathrm{mL}$ ) from Abbexa (Cambridge, United Kingdom).

All ELISA experiments were performed according to the instructions provided by the manufacture of each kit without any modification. All assays employed the quantitative sandwich enzyme immunoassay technique. The concentration of the antigens of interest (i.e., biomarkers) in serum samples was determined using a relative standard curve. Samples were measured in triplicate and the mean values were used in the analysis. The intra- and inter-assay coefficient of variance $(\mathrm{CV})$ were $<10.1$ and $12.3 \%$ respectively for COMP, $<6.5$ and $9.7 \%$ respectively for HA and each $<8 \%$ for PIICP.

\section{Statistical analysis}

Continuous variables with normal distribution were expressed as means \pm standard deviation (SD). Nonnormally distributed variables were expressed as medians and interquartile range (IQR). The normal distribution of continuous data was analyzed with the D'Agostino \& Pearson omnibus normality test. Significant differences in demographic data that followed Gaussian distribution were calculated using unpaired $t$-test or one-way ANOVA and adjusted by Tukey's multiple comparison method tests for multiple comparisons. The D'Agostino \& Pearson omnibus normality test was used to assess that the levels of biomarkers in serum samples of the total study population, as well as of each of the KL grade groups, were normally distributed, which they were not, and therefore nonparametric tests were subsequently used. Thus, biomarker concentrations between KL grade groups (OA severity) and pain groups (pain at rest and during walking) were compared using the Kruskal-Wallis $H$-test and followed by Dunn's multiple comparison test. The Mann-Whitney $U$-test was employed to compare the biomarker levels (pairwise comparison) in age groups (i.e., for healthy individuals and patients with EOA: $40-54$ years and $\geq 55$ years and patients with LOA: $50-69$ years and $\geq 70$ years) and gender groups.
Age grouping for healthy individuals and patients with EOA was carried out taking into account the mean age of participants in these groups, which was approximately 55 years while the mean age of the participants in the LOA was $\sim 70$ years (Table 1 ). The linear relationship of serum biomarkers with continuous variables [i.e., age, BMI, KL grade, and pain at rest (resting VAS) or during walking (walking VAS)] was carried out using Spearman's correlation coefficient. Correlations were classified as very weak (correlation coefficient $(r)<0.20)$, weak $(r=0.20-0.39)$, moderate $(r=0.40-0.59)$, strong $(r=0.60-0.79)$, or very strong $(r>0.80)$. A multiple linear regression analysis was also conducted to evaluate the serum biomarker levels and OA risk factors including age, BMI, KL grade, pain during resting and walking, familial OA, and occupational risk. Finally, ROC curve analysis was used to evaluate the diagnostic value of serum biomarker expression in patients with early or late OA. Other diagnostic parameters were also evaluated, including sensitivity, specificity, cut-off value, and area under the ROC curve (AUC) with a 95\% confidence interval $(\mathrm{CI})$, to assess the discrimination power of each biomarker. All reported $p$-values were two-tailed and $p$-values $<0.05$ were considered statistically significant. Statistical analysis was performed using GraphPad Prism (v.8.2, GraphPad Software Inc., San Diego, CA, USA). ROC curve analysis and calculation of cut-off point for PIICP were carried out using MedCalc Statistical Software version 19.2.6 (MedCalc Software, Ostend, Belgium).

\section{Results}

\section{Description of the study population}

The demographic and clinical characteristics of the study population are illustrated in Table 1 . To be included in the analysis, participants had to have a knee radiographical assessment for both knees and have replied to all questions of the questionnaires. The study involved 38 healthy overweight individuals (23 women, 15 men) at high risk of developing OA with a mean age $( \pm \mathrm{SD})$ of $50.7 \pm 6.3$ years and a mean BMI ( \pm SD) of $28.30 \pm 2.67 \mathrm{~kg} / \mathrm{m}^{2}$. The early kneeOA group consisted of 44 participants (31 women, 13 men). Mean age $( \pm$ SD) and mean BMI $( \pm$ SD) in this group was $52.4 \pm 5.6$ years and $27.02 \pm 4.27 \mathrm{~kg} / \mathrm{m}^{2}$, respectively. No statistically significant differences in age or BMI were observed between men and women in both the healthy and early OA groups. In this study, 130 patients (32 men and 98 women) with late (established) OA were also recruited. Patients with LOA were also divided into a mild group (KL grade of 2 in at least one knee, $n=35$ ), and a severe group (KL grade of 3 or 4 in at least one knee, $n=95$ ). Mean age and BMI $( \pm \mathrm{SD})$ in the total population of patients with LOA 
Table 1 Demographic data and characteristics of the subjects in this study

\begin{tabular}{|c|c|c|c|c|c|}
\hline \multirow[t]{2}{*}{ Variable } & \multirow[t]{2}{*}{ Healthy } & \multirow[t]{2}{*}{ EOA } & \multicolumn{3}{|l|}{ LOA } \\
\hline & & & Total & Mild $^{a}$ & Severe $^{b}$ \\
\hline n (\%) & 38 & 44 & 130 & 35 & 95 \\
\hline Men & $15(39.5)$ & $13(29.5)$ & $32(24.6)$ & $3(8.6)$ & $29(30.5)$ \\
\hline Women & $23(60.5)$ & $31(70.5)$ & $98(75.4)$ & $32(91.4)$ & $66(69.5)$ \\
\hline Age, years (range) & $50.7 \pm 6.3^{*}(39-62)$ & $52.4 \pm 5.6^{\#}(42-65)$ & $70.3 \pm 8.3(50-85)$ & $68.1 \pm 8.66(50-81)$ & $71.1 \pm 8.0(50-85)$ \\
\hline $\mathrm{BMI}, \mathrm{kg} / \mathrm{m}^{2}$ (Range) & $\begin{array}{l}28.30 \pm 2.67(25.04- \\
35.83)\end{array}$ & $\begin{array}{l}27.02 \pm 4.27^{\S, \neq} \\
(18.62-38.83)\end{array}$ & $\begin{array}{l}29.98 \pm 4.69(20.78- \\
46.88)\end{array}$ & $\begin{array}{l}28.73 \pm 4.59(20.90- \\
40.00)\end{array}$ & $\begin{array}{l}30.44 \pm 4.67 \\
(20.78-46.88)\end{array}$ \\
\hline \multicolumn{6}{|l|}{ Family history of OA, n (\%) } \\
\hline Yes & $26(68.4)$ & $34(77.3)$ & $78(60.0)$ & $25(71.4)$ & $53(55.8)$ \\
\hline No & $12(31.6)$ & $8(18.2)$ & $50(38.5)$ & $10(28.5)$ & $40(42.1)$ \\
\hline I do not know & 0 & $2(4.5)$ & $2(1.5)$ & 0 & $2(2.1)$ \\
\hline Occupational risk, n (\%) & $16(42.1)$ & $25(56.8)$ & $34(26.2)$ & $4(11.4)$ & $30(31.6)$ \\
\hline $\begin{array}{l}\text { Previous knee injury, } \\
\mathrm{n}(\%)\end{array}$ & $15(39.5)$ & $19(43.2)$ & $29(22.3)$ & $10(28.6)$ & $19(20.0)$ \\
\hline \multicolumn{6}{|l|}{ Left Knee } \\
\hline Pain at rest (0-10) & - & $2.3 \pm 1.5(0-8)$ & $4.6 \pm 2.3(0-9)$ & $4.2 \pm 2.0(0-8)$ & $4.8 \pm 2.9(0-9)$ \\
\hline Pain at walking $(0-10)$ & - & $3.6 \pm 2.4(0-10)$ & $6.0 \pm 2.3(0-10)$ & $5.8 \pm 1.9(1-9)$ & $6.0 \pm 2.4(0-10)$ \\
\hline \multicolumn{6}{|l|}{$\mathrm{KL}$ grade, $\mathrm{n}(\%)$} \\
\hline 0 & $38(100)$ & $42(95.5)$ & - & - & - \\
\hline 1 & - & $2(4.5)$ & $4(3.1)$ & 0 & $4(4.2)$ \\
\hline 2 & - & - & $42(32.3)$ & $35(100.0)$ & $7(7.4)$ \\
\hline 3 & - & - & $78(60.0)$ & - & $78(82.1)$ \\
\hline 4 & - & - & $6(4.6)$ & - & $6(6.3)$ \\
\hline \multicolumn{6}{|l|}{ Right Knee } \\
\hline Pain at rest (0-10) & - & $2.9 \pm 2.7(0-8)$ & $4.6 \pm 2.2(0-9)$ & $3.9 \pm 2.2(0-8)$ & $4.8 \pm 2.3(0-9)$ \\
\hline Pain at walking $(0-10)$ & - & $4.1 \pm 1.8(0-10)$ & $6.0 \pm 2.3(0-10)$ & $5.4 \pm 2.6(0-10)$ & $6.2 \pm 2.2(0-9)$ \\
\hline \multicolumn{6}{|l|}{ KL grade, $n(\%)$} \\
\hline 0 & $38(100)$ & $38(86.4)$ & - & - & - \\
\hline 1 & - & $6(13.6)$ & $10(7.7)$ & $1(2.9)$ & $9(9.5)$ \\
\hline 2 & - & - & 38 (29.2) & $34(97.1)$ & $4(4.2)$ \\
\hline 3 & - & - & $73(56.2)$ & - & $73(76.8)$ \\
\hline 4 & - & - & $9(6.9)$ & - & $9(9.5)$ \\
\hline
\end{tabular}

Except where indicated, data are presented as mean \pm standard deviation

$B M I$ Body mass index, OA Osteoarthritis, EOA Early OA, LOA Late OA, KL Kellgren and Lawrence grade

${ }^{a}$ Mild-LOA: KL of 2 in at least one knee; ${ }^{b}$ Severe-LOA: KL of 3 or 4 in at least one knee Differences among groups were compared by analysis of variance or Student's t-test ${ }^{*} p<0.0001$ : healthy group vs i) Total-LOA, ii) LOA-mild, and iii) LOA-severe OA groups \# $p<0.0001$ : EOA group vs i) LOA, ii) mild, and iii) severe OA groups

$\S p<0.01$ : EOA group vs LOA group

${ }^{*} p<0.001$ : EOA group vs Severe group

were $70.3 \pm 8.3$ years and $29.98 \pm 4.36 \mathrm{~kg} / \mathrm{m}^{2}$, respectively. No statistically significant differences in age or BMI were observed between men and women in these groups. KL scores ranged from 1 to 4 . The majority of the participants $(64.6 \%)$ had a KL score of $\geq 3$ while all OA patients had both knees affected, and 71 patients (54.6\%) had a KL score of $\geq 3$ in both knees. Also, there were no statistically significant differences in KL scores between the left and right knees in these groups. OA was graded $\mathrm{KL} \geq 3$ in 26 of $32(81 \%)$ men and 58 of 92 (59.2\%) women $(p>0.05)$. There were no statistically significant differences between men and women in pain scores $(p>0.05)$, except the pain score in the right knee $(p<0.01)$. There were also statistical differences in pain scores between patients with early- and lateOA $(p<0.01)$. In addition, there were no statistically 
significant differences in KL scores between the left and right knees $(p>0.05)$ in all groups as revealed by Student's $t$-test (data not shown).

\section{Assessment of prevalence of knee OA according to different definitions}

The prevalence of knee OA was assessed based on different definitions among participants per group, and all observations are presented in Table 2. Initially, we assessed the prevalence of knee OA using a 15-scale bilateral definition i.e., a combination of $\mathrm{KL}$ grades of both knees (0:0), (1:0), (1:1), .., (4:2), (4:3), (4:4), as illustrated in Table 2. However, according to this definition, some groups consisted of a small number of participants.
Therefore, we subsequently, assessed knee OA prevalence using a 9 -scale bilateral definition as previously described [44] i.e., the mean KL grade of both knees to allow linear associations (Table 2). The most prevalent mean KL grade was 0.0 followed by 3.0 and 2.0 accounting for $35.8,29.7$, and $16.0 \%$ of participants, respectively. The prevalence of knee OA according to this definition in the total population of this study as well as per group is summarized in Table 2. It should be pointed out that the main advantage of combining $\mathrm{KL}$ scores is that smaller group numbers are created (i.e., 15 using the combination of KL scores vs 9 using the bilateral mean KL scores) which enhances the reported confidence intervals [44]. Importantly, the KL grades of the knees are neither

Table 2 Prevalence of knee OA according to two different definitions

\begin{tabular}{|c|c|c|c|c|c|c|}
\hline \multicolumn{7}{|c|}{ Prevalence of knee OA based on a combination of KL grades } \\
\hline \multirow[t]{3}{*}{ Combination of $\mathrm{KL}$ grades } & \multicolumn{5}{|l|}{ Group, n } & \multirow{3}{*}{$\begin{array}{l}\text { Total } \\
\text { Population }\end{array}$} \\
\hline & Healthy & Early OA & Late OA & & & \\
\hline & & & Total & Mild & Severe & \\
\hline $0: 0$ & 38 & 38 & - & - & - & 76 \\
\hline $1: 0$ & - & 4 & - & - & - & 4 \\
\hline $1: 1$ & - & 2 & - & - & - & 2 \\
\hline $2: 0$ & - & - & - & - & - & 0 \\
\hline $2: 1$ & - & - & 1 & 1 & - & 1 \\
\hline $2: 2$ & - & - & 34 & 34 & - & 34 \\
\hline $3: 0$ & - & - & 0 & - & - & 0 \\
\hline $3: 1$ & - & - & 10 & - & 10 & 10 \\
\hline $3: 2$ & - & - & 10 & - & 10 & 10 \\
\hline $3: 3$ & - & - & 63 & - & 63 & 63 \\
\hline $4: 0$ & - & - & 0 & - & 0 & 0 \\
\hline $4: 1$ & - & - & 3 & - & 3 & 3 \\
\hline $4: 2$ & - & - & 1 & - & 1 & 1 \\
\hline $4: 3$ & - & - & 4 & - & 4 & 4 \\
\hline $4: 4$ & - & - & 4 & - & 4 & 4 \\
\hline Total & 38 & 44 & 130 & 35 & 95 & 212 \\
\hline \multicolumn{7}{|c|}{ Prevalence of knee OA based on the mean of $\mathrm{KL}$ grades } \\
\hline \multirow[t]{3}{*}{ Mean of KL grades } & \multicolumn{5}{|l|}{ Group, n } & \multirow{3}{*}{$\begin{array}{l}\text { Total } \\
\text { Population }\end{array}$} \\
\hline & Healthy & Early OA & Late OA & & & \\
\hline & & & Total & Mild & Severe & \\
\hline 0.0 & 38 & 38 & - & - & - & 76 \\
\hline 0.5 & - & 4 & - & - & - & 4 \\
\hline 1.0 & - & 2 & - & - & - & 2 \\
\hline 1.5 & - & - & 1 & 1 & - & 1 \\
\hline 2.0 & - & - & 44 & 34 & 10 & 44 \\
\hline 2.5 & - & - & 13 & - & 13 & 13 \\
\hline 3.0 & - & - & 64 & - & 64 & 64 \\
\hline 3.5 & - & - & 4 & - & 4 & 4 \\
\hline 4.0 & - & - & 4 & - & 4 & 4 \\
\hline Total & 38 & 44 & 130 & 35 & 95 & 212 \\
\hline
\end{tabular}

${ }^{\mathrm{a}}$ Total population $=$ health individuals + patients with early $\mathrm{OA}+$ total number of patients with late $\mathrm{OA}$ 
omitted (e.g., in several reports the KL grade that is used for analysis is the higher one in patients who have both knees affected) nor summarized [44]. Therefore, in this study, for analysis, the mean KL grades of both knees were used.

\section{Correlation of biomarker levels and OA severity}

The levels of the three biomarkers in the healthy group, EOA, and LOA groups as well as in patients with mildLOA and severe-LOA are illustrated in Fig. 1 (a-c). We initially compared the levels of the three biomarkers among healthy individuals, EOA, and LOA (total population) groups using the Kruskal Wallis and adjusted for multiple comparisons by Dunn's multiple comparisons method with an $\alpha$ of 0.05. As shown in Fig. 1a and Fig. 1b, respectively, median (IQR) SCOMP and sHA values were higher in the LOA group compared to the EAO and healthy group, but these differences did not reach statistical significance $(p>0.05)$. There were no statistically significant differences in the concentrations of sCOMP, sHA, and sPIICP between the healthy group and the EOA group (Fig. $1 \mathrm{a}-\mathrm{c}$ ). Interestingly, the sPIICP levels were significantly lower $(p<0.0001)$ in the LOA group compared to those in the healthy and EOA groups as resulted by Kruskal-Wallis test followed by Dunn's multiple comparisons (Fig. 1c).

We subsequently examined the differences in biomarker levels among healthy individuals, EOA, mildand severe-LOA groups using the Kruskal Wallis and adjusted for multiple comparisons by Dunn's multiple comparisons method with an $\alpha$ of 0.05 . Based on this classification of LOA, the median (IQR) sCOMP levels of $316.0(217.2-421.88) \mathrm{ng} / \mathrm{mL}$ in the severe-LOA group were significantly higher compared to those in the healthy [206.6 $(178.9-277.3) \mathrm{ng} / \mathrm{mL}, p<0.01]$ and the EOA group [178.8 (134.0-265.4) ng/mL, $p<0.0001$ ] (Fig. 1a).

However, there was no statistically significant difference $(p>0.05)$ in the sCOMP levels between the EOA group and i) healthy group and ii) severe-LOA group. Serum HA levels showed a trend of slight increase as OA severity increased, but these differences did not reach statistical significance $(p>0.05)$ (Fig. 1b). On the contrary, the sPIICP levels showed a trend of decreased values as OA severity increased (Fig. 1c). Median values (IQR) of sPIICP levels [703.4 $(593.0-922.8) \mathrm{pg} / \mathrm{mL}$ ] were significantly $(p<0.0001)$ higher in the healthy group compared to the mild-LOA group [179.1 (148.4-278.9) pg/mL] and severe-LOA group [177.6 (109.1-273.3) $\mathrm{pg} / \mathrm{mL}]$. There was also statistically significant difference $(p<0.0001)$ in sPIICP levels between the EOA group and the mild- LOA group as well as the severe-LOA group. There was no statistically significant difference $(p>0.05)$ in sPIICP levels between the health and EOA groups as well as between the mild and severe OA groups (Fig. 1c).

Spearman correlation was also employed to assess the collinearity of the biomarkers (Table 3). The results revealed that SCOMP correlated very weakly and negatively with sPIICP in the total population $(r=-0.184$, $p<0.01)$. In the healthy group, there were no correlations

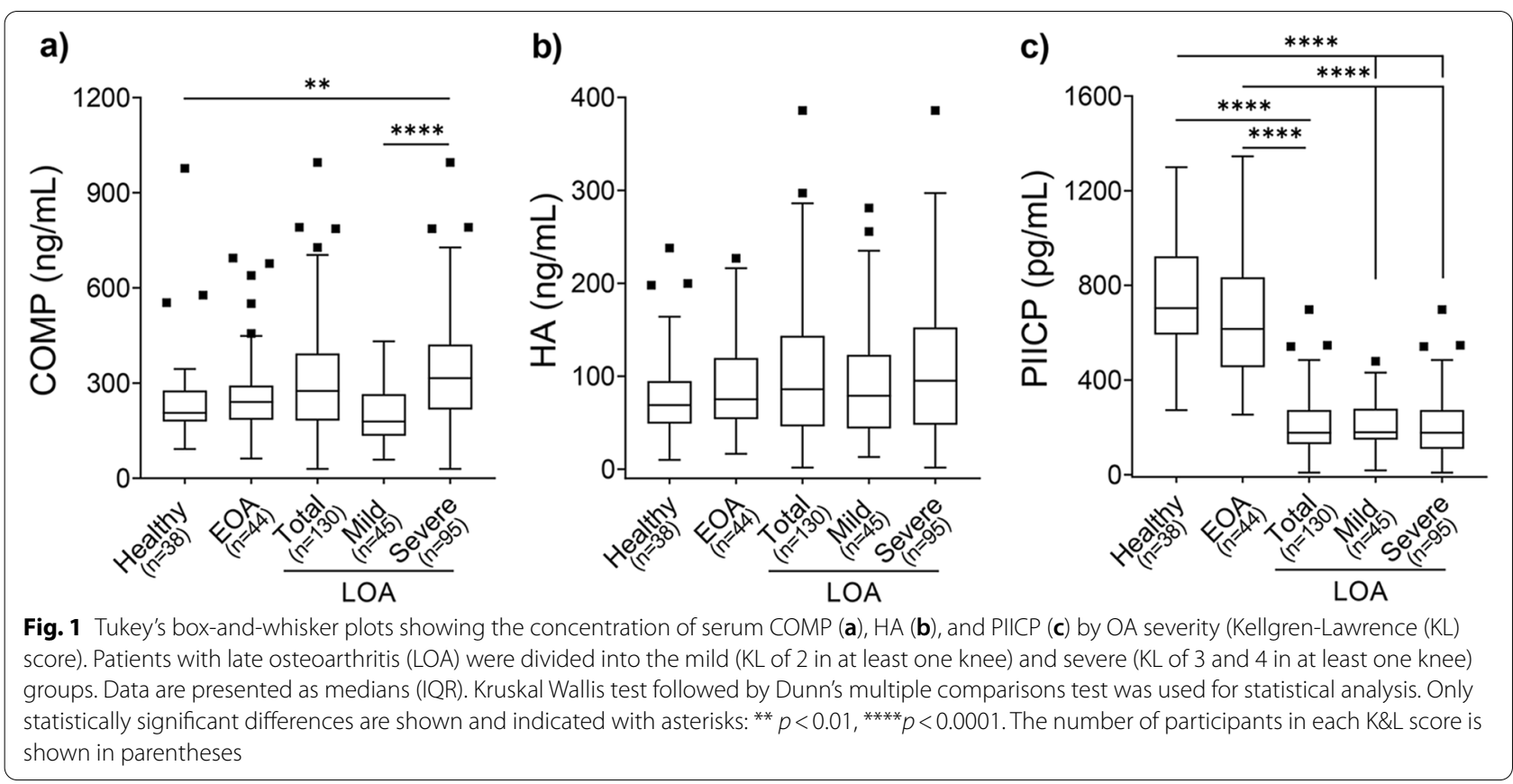


Table 3 Correlation between biomarker levels in the total population, healthy individuals, and patients with EOA or LOA

\begin{tabular}{|c|c|c|c|c|c|c|c|c|}
\hline \multirow[t]{2}{*}{ Biomarkers } & \multicolumn{2}{|c|}{ Total population } & \multicolumn{2}{|c|}{ Healthy } & \multicolumn{2}{|l|}{ EOA } & \multicolumn{2}{|l|}{ LOA } \\
\hline & $r$ & $p$ & $r$ & $p$ & $r$ & $p$ & $r$ & $p$ \\
\hline COMP vs HA & 0.121 & $>0.05$ & 0.072 & $>0.05$ & 0.017 & $>0.05$ & 0.177 & $<0.05$ \\
\hline COMP vs PIICP & -0.184 & $<0.01$ & 0.086 & $>0.05$ & 0.448 & $<0.01$ & -0.307 & $<0.0001$ \\
\hline PIICP vs HA & -0.030 & $>0.05$ & 0.050 & $>0.05$ & -0.117 & $>0.05$ & 0.123 & $>0.05$ \\
\hline
\end{tabular}

EOA Early Osteoarthritis, LOA Late Osteoarthritis. Correlation analysis between biomarker levels was performed using Spearman's rank correlation coefficient (r). All reported $p$-values are two-tailed. $p$-values $<0.05$ were considered statistically significant and indicated in bold

among the three biomarkers. Interestingly, in the EOA group, sCOMP was positively and moderately correlated with sPIICP $(r=0.448, p<0.01)$. In the LOA group, sCOMP was very weakly and positively correlated with sHA $(r=0.177, p<0.05)$ while a weak and negative correlation was obtained between SCOMP and PIICP $(r=-0.307, p<0.01)$.

\section{Correlation between biomarker levels and knee pain}

Patients were divided into four groups according to the bilateral mean knee pain intensity both at rest

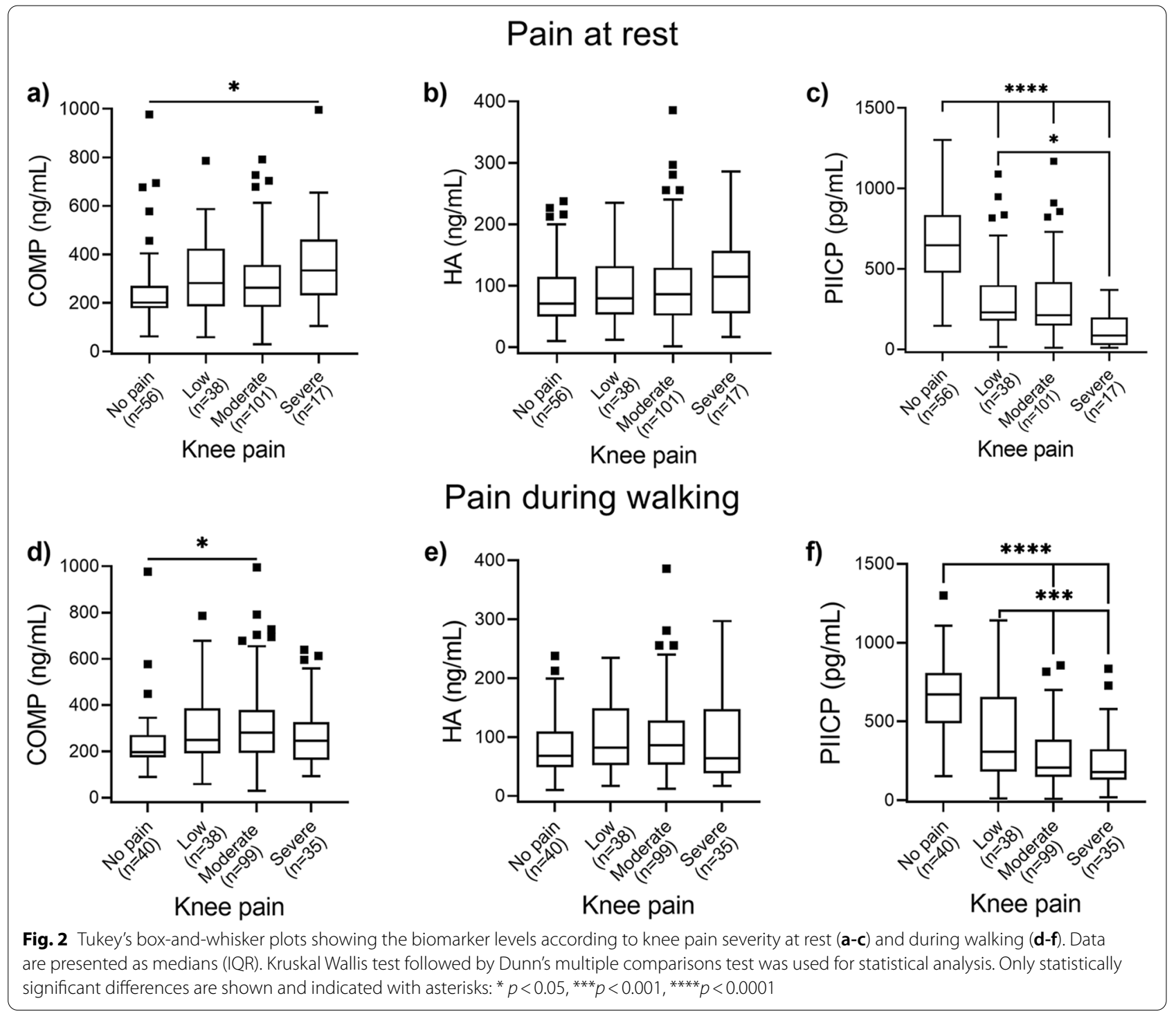


(Fig. 2 a-c) and during walking (Fig. 2 d-f). The presence of low or moderate pain did not affect the levels of sCOMP, however, the levels of this biomarker were significantly higher $(p<0.05)$ in the group with severe pain compared to those without knee pain at rest (Fig. 2a). Also, the levels of sCOMP were higher in the group with moderate pain during walking $(p<0.05)$ compared to the group without pain (Fig. 2d). There was no significant difference $(p>0.05)$ in sHA levels among the four pain groups both at rest (Fig. 2b) and during walking (Fig. 2e). The levels of sPIICP were significantly higher in individuals without knee pain at rest compared to those with low, moderate, and severe knee pain $(p<0.0001)$ (Fig. 2c). There was also statistically significant difference in sPIICP levels between the low- and severe-pain at rest groups $(p<0.05)$. There was statistically significant difference in the SPIICP levels between the group without pain during walking and i) the group with moderate $(p<0.0001)$ and ii) severe pain group $(p<0.0001)$. There was also statistically significant difference in sPIICP levels between the group with low pain during walking and both the moderate and severe pain groups $(p<0.001)$ (Fig. 2f).

\section{Correlation of biomarker levels and clinical parameters}

Spearman's correlation coefficient was calculated to examine the association between the levels of biomarkers in serum and risk factors (continuous factors) for OA including age, BMI, KL grade (bilateral mean value), as well as pain at rest (resting VAS), and walking (walking VAS) (Table 4). The levels of sCOMP increased with age and BMI and were weakly correlated with KL grade. Furthermore, sCOMP levels were very weakly correlated with pain at rest, but they were not correlated with pain during walking. Serum HA levels were weakly correlated only with age and very weakly correlated with KL grade. Interestingly, sPIICP levels were significantly and negatively correlated to OA risk factors such as age and BMI, as well as with $\mathrm{KL}$ grade and bilateral mean pain intensity at rest and during walking (Table 4). It should be noted that the negative coefficient values indicated that the variables are inversely related (i.e., as the value of one variable increases, the value of the other tends to decrease).

Moreover, we performed a multiple linear regression analysis to identify potential associations among the parameters of age, BMI, pain, KL grade, gender, familiar $\mathrm{OA}$, and occupational risk with each of the biomarkers (Table 5). The results revealed that serum COMP and HA were associated with age and occupational risk, whereas $\mathrm{BMI}$, gender, $\mathrm{KL}$ score, pain at resting and during walking as well as with a previous knee injury and familial OA exerted no statistically significant influence on the levels of serum COMP and HA. Interestingly, the levels of serum PIICP were independently associated with KL grades $(p<0.0001)$, while the other factors did not have a statistical relationship with the levels of this biomarker.

The diagnostic efficiency of the three biomarkers i.e., their ability to differentiate patients with EOA or LOA from healthy individuals, was further evaluated by the sensitivity, specificity, Youden index, and the area under the ROC curve (Table 6). The three serum biomarkers have limited diagnostic potential in early knee osteoarthritis because the reliability of the cutoff values for the development of EOA was low (AUC <0.6; Table 6). The ROC curves for the three serum biomarkers for EOA and LOA are illustrated in Fig. 3.

Interestingly, our analysis revealed that among three serum markers, PIICP had the highest AUC value (0.980, 95\%CI: 0.945-0.995; $p<0.0001)$ for LOA diagnosis, followed by COMP (Table 6). Therefore, according to our analysis, sPIICP could discriminate between patients with LOA and healthy individuals, with a sensitivity of $92.6 \%$ and specificity of $92.1 \%$. The cut-off value of PIICP for LOA diagnosis was $465.4 \mathrm{pg} / \mathrm{mL}$.

Table 4 Association of biomarker levels with age, BMI, KL grade, and knee pain

\begin{tabular}{|c|c|c|c|c|c|c|}
\hline \multirow[t]{3}{*}{ Variable } & \multicolumn{6}{|c|}{ Biomarker } \\
\hline & \multicolumn{2}{|l|}{ sCOMP } & \multicolumn{2}{|l|}{ sHA } & \multicolumn{2}{|l|}{ sPIICP } \\
\hline & $r$ & $p$-value & $r$ & $p$-value & $r$ & $p$-value \\
\hline Age & 0.2574 & 0.0002 & 0.2523 & 0.0002 & -0.5807 & $<0.0001$ \\
\hline BMI & 0.2150 & 0.0016 & -0.0921 & 0.1818 & -0.2225 & 0.0011 \\
\hline KL grade & 0.2417 & 0.0004 & 0.1399 & 0.0419 & -0.6776 & $<0.0001$ \\
\hline Resting VAS & 0.1607 & 0.0192 & 0.0263 & 0.7029 & -0.4442 & $<0.0001$ \\
\hline Walking VAS & 0.0624 & 0.3657 & 0.0059 & 0.9314 & -0.4184 & $<0.0001$ \\
\hline
\end{tabular}

Correlation analysis between biomarker levels and the variables was performed using Spearman's rank correlation coefficient (r). All reported $p$-values are two-tailed. $p$-values $<0.05$ were considered statistically significant and indicated in bold 
Table 5 Multiple linear regression for the assessment of OA risk factors on the levels of the three serum biomarkers

\begin{tabular}{|c|c|c|c|c|c|c|c|c|c|c|c|c|}
\hline \multirow[t]{2}{*}{ Variable } & \multicolumn{4}{|l|}{ sCOMPa } & \multicolumn{4}{|l|}{$s H A^{b}$} & \multicolumn{4}{|l|}{ sPIICPc } \\
\hline & Est & SE & $t$ & $p$-value & Est & SE & $\mathbf{t}$ & $p$-value & Est & SE & $\mathbf{t}$ & $p$-value \\
\hline (Constant) & -14.73 & 103.4 & 0.143 & 0.8868 & 35.95 & 42.51 & 0.8458 & 0.3987 & 695.4 & 136.2 & 5.106 & $<0.0001$ \\
\hline Age (per year) & 2.865 & 1.421 & 2.017 & 0.0450 & 1.773 & 0.584 & 3.035 & 0.0027 & -0.622 & 1.872 & 0.3323 & 0.7400 \\
\hline BMI (per $\left.1 \mathrm{~kg} / \mathrm{m}^{2}\right)$ & 4.191 & 2.502 & 1.675 & 0.0955 & -1.663 & 1.029 & 1.616 & 0.1077 & 1.228 & 3.297 & 0.3725 & 0.7099 \\
\hline Gender & -30.76 & 26.32 & 1.168 & 0.2440 & -4.817 & 10.83 & 0.445 & 0.6569 & -62.96 & 34.69 & 1.815 & 0.0710 \\
\hline $\mathrm{KL}$ grade & 15.08 & 14.36 & 1.050 & 0.2950 & 2.124 & 5.907 & 0.359 & 0.7195 & -163.5 & 18.92 & 8.639 & $<0.0001$ \\
\hline Resting VAS & 0.843 & 6.409 & 0.132 & 0.8955 & -2.250 & 2.636 & 0.8535 & 0.3944 & 0.321 & 8.445 & 0.0381 & 0.9697 \\
\hline Walking VAS & -7.532 & 5.840 & 1.290 & 0.1986 & -0.615 & 2.402 & 0.2561 & 0.7981 & 2.459 & 7.695 & 0.3195 & 0.7496 \\
\hline $\begin{array}{l}\text { Previous knee } \\
\text { Injury }\end{array}$ & 20.49 & 24.21 & 0.846 & 0.3984 & 5.480 & 9.956 & 0.5504 & 0.5826 & 6.553 & 31.90 & 0.2054 & 0.8374 \\
\hline $\begin{array}{l}\text { Occupational } \\
\text { Risk }\end{array}$ & 78.31 & 25.64 & 3.054 & 0.0026 & 21.30 & 10.54 & 2.020 & 0.0447 & 26.83 & 33.78 & 0.7941 & 0.4281 \\
\hline Familial OA & 1.902 & 22.94 & 0.083 & 0.9340 & -2.966 & 9.436 & 0.3144 & 0.7536 & -10.28 & 30.23 & 0.3402 & 0.7341 \\
\hline
\end{tabular}

$p$-values $<0.05$ are consider significant and indicated in bold

${ }^{a} R^{2}=0.153$, model ANOVA: $F=4.05, p<0.0001$

${ }^{\mathrm{b}} \mathrm{R}^{2}=0.112$, model ANOVA: $\mathrm{F}=2.84, p<0.001$

${ }^{c} R^{2}=0.560$, model ANOVA: $F=26.81, p<0.0001$

Table 6 RoC Curve Analysis for the diagnosis of patients with EOA or LOA

\begin{tabular}{|c|c|c|c|c|c|c|}
\hline \multirow[t]{2}{*}{ Parameter } & \multicolumn{3}{|l|}{ EOA } & \multicolumn{3}{|l|}{ LOA } \\
\hline & COMP & HA & PIICP & COMP & HA & PIICP \\
\hline Sensitivity & 47.7 & 50.0 & 52.7 & 49.2 & 47.5 & 96.2 \\
\hline Specificity & 68.4 & 65.8 & 73.7 & 78.9 & 76.3 & 92.1 \\
\hline Youden Index & 0.162 & 0.158 & 0.2596 & 0.282 & 0.240 & 0.8826 \\
\hline AUC & 0.558 & 0.555 & 0.596 & 0.609 & 0.578 & 0.980 \\
\hline$p$-value & $>0.05$ & $>0.05$ & $>0.05$ & $<0.05$ & $>0.05$ & $<0.0001$ \\
\hline $95 \% \mathrm{Cl}$ & $0.444-0.668$ & $0.441-0.665$ & $0.482-0.703$ & $0.531-0.684$ & $0.500-0.654$ & $0.945-0.995$ \\
\hline
\end{tabular}

EOA Early Osteoarthritis, LOA Late Osteoarthritis, AUC Area Under Curve, CI Confidence interval
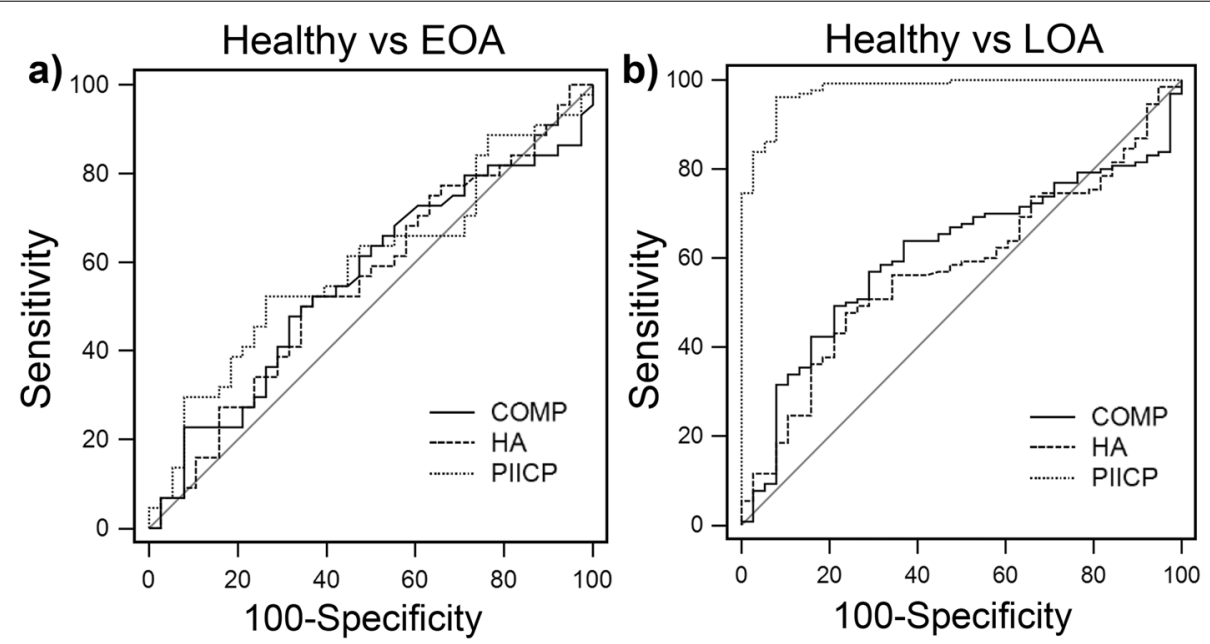

Fig. 3 ROC curves for the three individual biomarkers (COMP, HA, and PIIICP) in healthy individuals and patients with early (a) and late (b) osteoarthritis 


\section{Subgroup analysis}

We subsequently divided the participants of the healthy group and EOA into 2 age categories namely age $40-54$ years ( $n=28$ in both groups) and age $\geq 55$ years groups ( $n=10$ in healthy group and $n=16$ in EOA group). Likewise, we divided the participants with established (late) OA into two age categories namely age 50-69years $(n=57)$ and age $\geq 70$ years $(n=73)$ groups. Patients with mild- and severe- LOA were also divided into the 50-69years $(n=57)$ and age $\geq 70$ years $(n=73)$ groups (Fig. 4 a-c).

As illustrated in Fig. 4a, in patients with EOA, sCOMP levels were significantly lower $(p<0.05)$ in the age $40-54$ years group compared to the age $\geq 55$ years group. Median (IQR) values of sHA were significantly higher $(p<0.05)$ in the age $\geq 70$ years group compared to the age $50-69$ years group in both the total population of patients with LOA as wells as in patients with mild-LOA (Fig. 4b).
There was no statistically significant difference in sPIICP levels among all groups (Fig. 4c).

Spearman correlation analysis was then used to assess the correlation between serum biomarkers and the age of participants in the different groups (Table 7). In the healthy and EOA groups, there was a weak correlation between sCOMP levels and the age of participants. In the total population of patients with LOA, there was a weak correlation between the levels of SCOMP and SHA and the age of participants. Interestingly, in the mild-LOA group, there was a moderate correlation between the sHA levels and the age of patients, while in the severeLOA group a weak correlation between the sCOMP levels and the age of participants was observed.

Comparison between genders (Fig. 5) by the MannWhitney $U$ test revealed that the levels of sCOMP were significantly higher in men compared to women in the
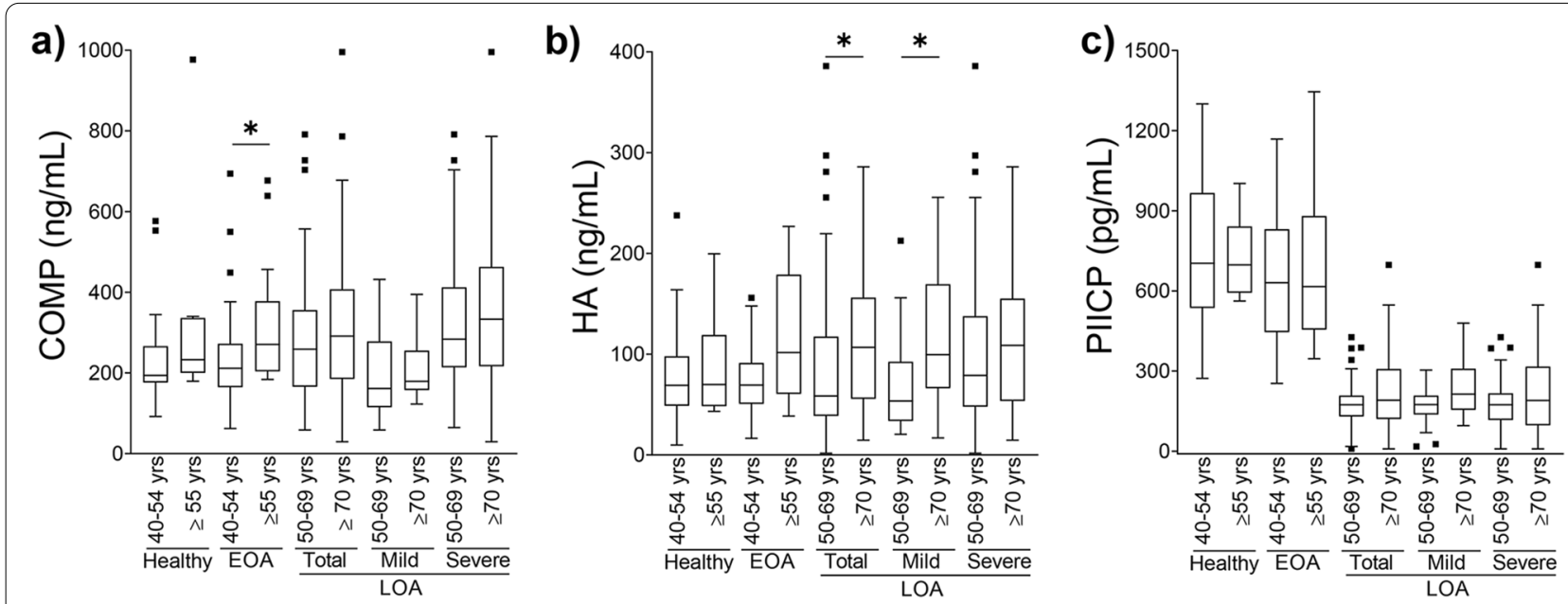

Fig. 4 Tukey's box-and-whisker plots showing the differences in the levels of serum COMP (a), HA (b), and PIICP (c) between the age 40-54years and age $\geq 55$ years groups in healthy participants and early osteoarthritis (EOA) patients and the age 50-69years and age $\geq 70$ years groups in patients with late osteoarthritis (LOA). Patients with LOA were also divided into the mild (KL of 2 in at least one knee) and severe (KL of 3 or 4 in at least one knee) groups. Data are presented as medians (IQR). Mann-Whitney $U$ test was used for pairwise statistical analysis. Only statistically significant differences are shown and indicated with asterisks: ${ }^{*} p<0.05$

Table 7 Correlation of biomarkers with the age of healthy individuals and patients with EOA and LOA

\begin{tabular}{|c|c|c|c|c|c|c|c|c|c|c|}
\hline \multirow[t]{3}{*}{ Biomarkers } & \multirow{2}{*}{\multicolumn{2}{|c|}{ Healthy }} & \multirow{2}{*}{\multicolumn{2}{|c|}{ EOA }} & \multicolumn{6}{|l|}{ LOA } \\
\hline & & & & & \multicolumn{2}{|l|}{ Total } & \multicolumn{2}{|l|}{ Mild } & \multicolumn{2}{|l|}{ Severe } \\
\hline & $r$ & $p$ & $r$ & $p$ & $r$ & $p$ & $r$ & $p$ & $r$ & $p$ \\
\hline COMP & 0.349 & $<0.05$ & 0.379 & $<0.05$ & 0.218 & $<0.05$ & 0.187 & $>0.05$ & 0.206 & $<0.05$ \\
\hline $\mathrm{HA}$ & 0.230 & $>0.05$ & 0.295 & $>0.05$ & 0.268 & $<0.01$ & 0.427 & $<0.05$ & 0.187 & $>0.05$ \\
\hline PIICP & 0.026 & $>0.05$ & 0.122 & $>0.05$ & 0.058 & $>0.05$ & 0.138 & $>0.05$ & -0.012 & $>0.05$ \\
\hline
\end{tabular}

EOA Early Osteoarthritis, LOA Late Osteoarthritis

Correlation analysis between biomarker levels and age of the participants was performed using Spearman's rank correlation coefficient ( $r$ ). All reported $p$-values are two-tailed. $p$-values $<0.05$ were considered statistically significant and indicated in bold 

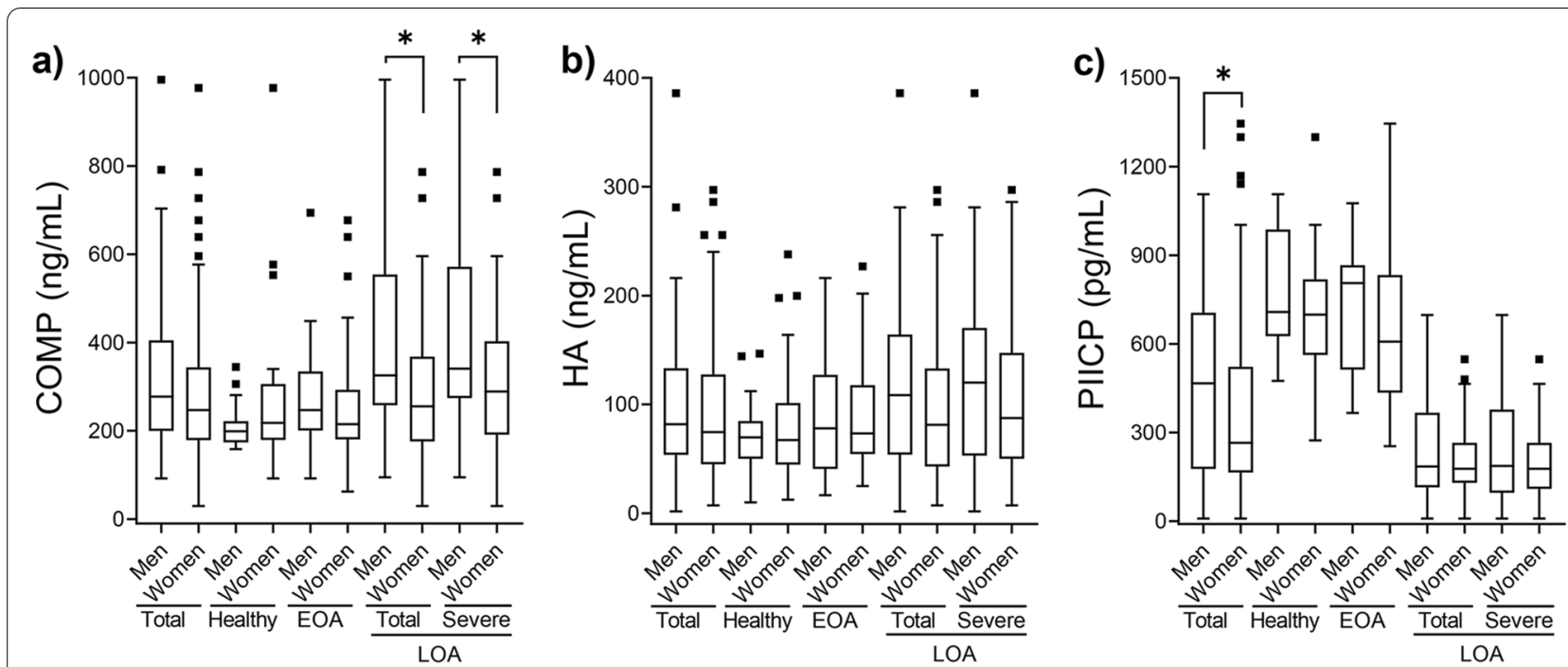

Fig. 5 Tukey's box-and-whisker plots showing the differences in the levels of serum COMP (a), HA (b), and PIICP (c) between men and women in the total population as well as in the healthy group and the groups of early osteoarthritis (EOA) and late osteoarthritis (LOA). Patients with LOA were divided into the mild (KL of 2 in at least one knee) and severe (KL of 3 or 4 in at least one knee) groups. Data are presented as medians (IQR). Mann-Whitney $U$ test was used for pairwise statistical analysis. Only statistically significant differences are shown and indicated with asterisks: * $p<0.05$

total population of patients with LOA (mild and severe) as well as in the severe-LOA group (Fig. 5a). There was no statistically significant difference in sHA levels between men and women in all groups as shown in Fig. 5b. The levels of sPIICP were significantly lower $(p<0.05)$ in females compared to males in the total population of participants (Fig. 5c). It should be noted that statistical analysis of the mild-LOA group was not performed because of the small number of male participants in this group.

\section{Discussion}

This study aimed to examine whether there are differences in the levels of two cartilage biomarkers (COMP and PIICP) and a synovitis biomarker (HA) among overweight healthy individuals, patients with EOA, and patients with LOA and examined whether the levels of these biomarkers in the three groups are associated with OA severity, knee pain at rest and during walking as well as with other OA risk factors. It has been generally accepted that a KL grade of 2 is the cut-off for defining radiographic knee OA features [45]. Therefore, in this study, we divided the patients with established OA into the mild group (KL grade of 2 in at least one knee) and severe group (KL grade of 3 or 4 in at least one knee). Because the majority of the patients in the LOA group had both knees affected, we used a 9-scale bilateral definition, i.e., the mean KL grade of both knees, to assess the severity of knee OA (Table 2).
In the current study, we demonstrate a clear correlation between the markers of cartilage synthesis (PIICP) and the severity of OA. Importantly, ROC analysis revealed that the AUC was 0.980, (95\% CI: 0.945-0.995; $p<0.001$ ), indicating high diagnostic accuracy of sPIICP i.e., this biomarker is suitable for the differentiation of healthy individuals at high risk of developing OA from patents with LOA (Fig. 3 and Table 6). It should be pointed out that our analysis revealed that SPIICP is not a suitable biomarker for the diagnosis of EOA. Moreover, our results revealed that SPIICP and sCOMP can detect pain-associated differences in patients regardless of the OA severity. The most significant finding of this work was that reduced serum levels of PIICP, which is one biomarker of cartilage synthesis, in patients with established OA were correlated with knee OA risk factors such as age and obesity. Type II collagen is a main component of the cartilage matrix that is synthesized by chondrocytes and its synthesis and breakdown are linked to cartilage metabolism. In detail, type II collagen is synthesized as a premature protein consists of three extra domains: a signal peptide, the procollagen type II N-terminal propeptide (PIINP), and the procollagen type II C-terminal propeptide (PIICP). These propeptides are cleaved off and released into biological fluids (e.g., blood, urine) during maturation [46]. Thus, the levels of these peptides in biological fluids reflect type II collagen synthesis and these two components could be used as markers to monitor the rate of OA progression [15]. It has also been 
demonstrated that PIICP levels in the joint fluid might be a prognostic marker for early OA in the knee as the concentration of PIICP was found to correlate with risk factors including obesity and varus knee alignment [15]. Post-traumatic damage of cartilage and periarticular tissues may also contribute to the development of OA [47] while obesity has been identified as a risk factor for the development of OA [48]. Our analysis also revealed that sPIICP concentration is also correlated with obesity and associated with occupational risk. In patients with severe OA, we found decreased levels of sPIICP compared to healthy individuals and patients with early $\mathrm{OA}$ and these findings are well in agreement with previous studies [31]. Another interesting result in this study was that the alterations in SPIICP levels are associated not only with the severity of knee OA but also with knee pain (Table 4). The mean levels of sPIICP in the groups with mild OA and severe $\mathrm{OA}$ were more than 2.5-fold lower than those in the healthy and EOA groups (Fig. 1c) suggesting that this decrease was not solely driven by factors such as age and obesity that are also correlated with the PIICP levels (Table 3). Our results are in good agreement with previous studies suggesting that PIICP concentrations in biological fluids might be an ideal prognostic marker for OA in the knee as the level of PIICP was found to correlate with risk factors such as obesity [7]. The progression of joint damage in OA is likely to result from an imbalance between cartilage degradation and synthesis processes and it has been proposed that quantifying the procollagen peptides in biological fluids could result in a better understanding of OA disease pathology and would provide means for evaluation of anabolic disease-modifying OA drugs [46]. Furthermore, because PIICP is released only during the synthesis of the new molecules, its production is known to reflect the rate of type II collagen synthesis and cartilage metabolism [49]. It was previously reported that both synovial fluid and serum levels of PIICP were increased in individuals with uncertain knee OA (KL grade of 0 or 1) [32]. On the contrary, the serum levels of PIICP were decreased in patients with earlystage of knee OA (K/L grade 2), where the radiographic joint space narrowing became clear [32]. A decrease of sPIICP levels $[19,31]$ and changes in the ratios of cartilage collagen degradation (C-terminal neopeptide/C2C and telopeptide fragment of collagen type-II/CTX-II) and synthesis (PIICP) markers $[15,19,50]$ were also reported with the onset of knee OA (KL grade 2 vs KL grade 1).

Interestingly, we also found a weak correlation between sCOMP levels and OA severity (KL grade), age, and BMI (Table 4). Regression analysis also revealed a correlation between sCOMP levels and i) age and ii) occupational risk (Table 5). COMP is a pentameric non-collagen protein related to the thrombospondin family, and also a constituent of articular cartilage and several studies have demonstrated that sCOMP is elevated in OA and after knee injury [51, 52] and therefore it might have some value as a diagnostic and/or prognostic marker of knee OA $[52,53]$. The majority of the previous studies investigating sCOMP as a biomarker for knee OA have shown that the levels of this biomarker are associated with structural and metabolic changes in OA [54]. Some studies investigating the association of sCOMP levels with clinical symptoms have shown inconsistent results [55]. Our analysis revealed that the level of COMP in serum is significantly higher in patients with severe OA (KL grade of 3 or 4) compare to that in the healthy group. Interestingly, our results also demonstrated that the serum level of COMP is also significantly higher in the severe OA group compared to that in the mild OA group, highlighting its potential as a prognostic biomarker as well as a biomarker for monitoring the progression of the disease. Elevated levels of sCOMP have been associated with knee pain [56]. Interestingly it has also been demonstrated that serum COMP levels are increased in patients with pain in the knees and without any radiological abnormalities, indicating early cartilage damage in these patients compared with healthy individuals [57]. In another study, the concertation of COMP in serum has been correlated with pain score but not OA severity (radiological grading), while it also negatively correlated with the progression of the disease. Thus, COMP levels can also be used as a prognostic marker to predict patients at risk of rapid progression [20].

In contrast to previous findings $[19,45,58]$, we could not demonstrate significant associations between sHA levels and clinical parameters including KL grade $(r=0.1399)$ and pain at rest $(r=0.0263)$ and during walking $(r=0.0059)$ (Table 4). However, in the multiple regression analysis sHA was correlated only with the KL grade (Table 5). Synovitis plays a vital role in the onset of OA [59] through the production of HA and pro-inflammatory cytokines including tumor necrosis factor- $\alpha$ and interleukin $1 \beta$. These cytokines induce the production of matrix metalloproteinase by fibroblasts resulting in the degradation of the articular cartilage matrix [60]. Previous studies have studied the association of sHA with the progression of knee OA [19, 61-63] (see also [45] and references cited therein), however, the data were limited. Sasaki et al. reported the correlation of sHA and radiographic progression of knee OA in a more general population including healthy individuals and patients with both early- and severe- knee OA for 5 years [45]. Even though the levels of sHA were positively correlated with KL grade progression the patients included in the study by Sasaki et al. were only middle-aged women and young individuals at very low risk of developing knee OA [45]. 
To better evaluate the role of HA in the development and progression of knee $\mathrm{OA}$ a wider range of ages may be needed. It has been previously reported that the sHA concentration might be also a specific biomarker for OA of other joints including the lumbar spine [45]. It has also been reported by Inoue et al. [64] that the correlation between sHA levels and OA was higher in patients with knee OA compared to those with hip OA or hand OA. However, sHA levels were increased with age because of an impaired ability of older individuals to metabolize HA [65]. Furthermore, other factors may contribute to the elevated sHA concentration in the elderly population such as hepatic failure, renal failure, and rheumatoid arthritis (RA) [66]. Therefore, patients suffering from hepatic and renal failure as well as from RA were excluded from this study allowing us to evaluate the correlation of sHA concentration and severity of knee OA more accurately. The lack of significant associations between HA levels and OA stage/severity suggests that this biomarker may not be a sensitive measure of the role of bone turnover in the progress of OA. Taken together the result of this study and those of others [33] indicate that sHA levels might not be an ideal biomarker for monitoring the progression of $\mathrm{OA}$ in elderly people.

Our analysis also revealed that there were no statistically significant differences in SCOMP and sHA levels between men and women in the total population; however, the sPIICP levels were statistically different $(p<0.05)$. Moreover, biomarker levels between males and female groups of identical OA severity (KL scores) revealed that the levels of sCOMP and sHA levels were higher in females compared to those in males in some but not all KL score groups, but the levels of sPIICP were found to be higher in males compared to females in the majority of $K \mathrm{~L}$ grade groups suggesting the association of these markers with the female gender in OA [67-69], and also warrants further research on the potential relevance of gender as an isolated factor for the use of these biomarkers in OA in the context of structural features. Females are at a higher risk of knee OA while several epidemiological studies revealed that the prevalence of knee OA in women is higher than in men [6].

\section{Limitations}

This study has some limitations. The recruitment of individuals has been carried out in two different research centers in two different countries (Spain and Cyprus) and thus we compared cohorts that differ not only in knee OA severity or presence (i.e. healthy individual and patients with EOA Spain, vs patients with LOA from Cyprus), but also in other potentially important aspects (e.g., recruitment process, assessment of knee OA, etc.) apart from the actual disease criteria. This also includes the risk of cluster bias as the two counties have significant differences in population, geography, and healthcare system, however, this work was part of a European project involving the recruitment of individuals from two different countries (i.e. Spain and Cyprus). Despite this, we managed to follow the same protocols for the recruitment of patients and the same standard operation processes for blood collection, handling, and storage as well as for the determination of the concentration of serum biomarkers. The patient number of our study was relatively small, while the sample size was not appropriately powered and thus further studies with larger participant numbers and properly powered are needed. The study was also limited by the fact that no control group (i.e., healthy non-obese young individuals) was included. Therefore, a comparison of biomarker and pain levels between healthy controls and our study population was not carried out in our analysis. All patients with established OA had KL scores ranging from 2 to 4 and suffered from clinically relevant advanced knee OA and thus they underwent knee replacement surgery. Thus, the reported biomarker levels represent the bone and cartilage turnover status of patients requiring surgery mainly due to functional/pain limitations and/or OAinduced symptoms. It should also be noted that the majority of patients with established (late) OA (mild $\mathrm{OA}$ and severe OA groups) suffered from bilateral knee OA. It has been proposed that the presence of unilateral OA may influence patient-reported physical function impairment more than bilateral OA [70]. Another limitation of our study was that we determined biomarker levels using ELISA, which may introduce both systematic and random errors. Moreover, while successful validation of a biomarker via ELISA depends on several factors including patient selection, collection methods, assay selection/handling, and stability of the marker. Nevertheless, we developed our SOPs for the determination of biomarkers levels via ELISA to minimize potential errors.

In this study, we were only able to measure the concentration of sCOMP, sPIICP, and sHA despite other biomarkers such as PIIANP [39], C-telopeptide of type II collagen (CTX-II) [67, 71], and synovial cytokines [72], and others [19] have been reported. Moreover, there was a statistical limitation because the relationship between each of the three biomarkers and the severity of OA and other OA risk factors was assessed using two tests, linear regression analysis, and Spearman's Correlation test. We did not use other analysis methods, e.g., multivariate regression analysis, because we did not want to statistically overestimate. Biomarker levels might be influenced by other factors, including age, obesity, gender, and disease stage. 


\section{Conclusions}

Despite the aforementioned limitations, the results of this work based on a general population consisting of healthy individuals at high risk of developing OA, patients with early OA as well as patients with established OA, demonstrated that the sPIICP concentration is strongly associated with the severity of knee $\mathrm{OA}$, knee pain both at resting and during walking and OA risk factors. Importantly our study highlights the potential of PIICP as a serum diagnostic biomarker for osteoarthritis and may facilitate diagnosis and monitoring the progression of the disease with relatively high sensitivity and specificity while the cutoff value of PIICP concentration predictive of the progression of OA was estimated. Further studies on the biomarkers that we examined here and the inclusion of additional biomarkers of the bone and cartilage turnover, in larger groups, are needed to make the results of this study more reliable and objective.

\section{Abbreviations}

AUC: Area Under Curve; BMI: Body mass index; COMP: Cartilage oligomeric matrix protein; EOA: Early osteoarthritis; HA: Hyaluronan; IQR: Interquartile range; K\&L: Kellgren and Lawrence; LOA: Late osteoarthritis; OA: Osteoarthritis; PIICP: Procollagen type II C-terminal propeptide; SD: Standard deviation.

\section{Acknowledgements}

Not applicable.

\section{Authors' contributions}

CP, AA-C, RM, CG, and KF conceptualized and design the study. AA-C, EV-C, $C C, M C, A E C, A K, A L, V M-L$, IP and $A C$ acquired the data. CP, AA-C, RM, CG, and $\mathrm{KF}$ performed the data analysis. CP and KF drafted the manuscript. All authors interpreted the data, critically revised the manuscript, and approved the final version of the manuscript.

\section{Funding}

The present study was funded by the European Community's H2020 programme under grant Nr. 77159.

\section{Availability of data and materials}

The data used to support the findings of this study will be available with the request to the corresponding authors.

\section{Declarations}

\section{Ethics approval and consent to participate}

The study was conducted according to the guidelines of the Declaration of Helsinki, and approved by the Cyprus National Bioethics Committee (EEBK) $\mathrm{E} \Pi / 2018 / 19)$ and the Ethics Committee on Drug Research of the University and Polytechnic Hospital La Fe. All participants gave their informed and written consent to participate in the study.

\section{Consent for publication}

Not applicable.

\section{Competing interests}

The authors declare that they have no competing interests.

\section{Author details}

1 Department of Life and Health Sciences, School of Sciences and Engineering, University of Nicosia, 2417 Nicosia, Cyprus. ${ }^{2}$ Physical Medicine \& Rehabilitation
Department, Hospital La Fe, 46026 Valencia, Spain. ${ }^{3}$ Health Research Institute La Fe, 46026 Valencia, Spain. ${ }^{4}$ Drug Development Area, Health \& Biomedicine Department, LEITAT Technological Centre, Parc Científic de Barcelona, 08028 Barcelona, Spain. ${ }^{5}$ Apollonion Hospital, 2054 Nicosia, Cyprus. ${ }^{6}$ Medical School, University of Nicosia, 2408 Nicosia, Cyprus.

Received: 15 October 2021 Accepted: 15 February 2022 Published online: 02 March 2022

\section{References}

1. Liu Q, Niu J, Li H, Ke Y, Li R, Zhang Y, et al. Knee symptomatic osteoarthritis, walking disability, NSAIDs use and all-cause mortality: population-based Wuchuan osteoarthritis study. Sci Rep. 2017;7(1):3309.

2. Geurts J, Jurić D, Müller M, Schären S, Netzer C. Novel exvivo human osteochondral explant model of knee and spine osteoarthritis enables assessment of inflammatory and drug treatment responses. Int J Mol Sci. 2018;19(5):1314.

3. Loeser RF, Goldring SR, Scanzello CR, Goldring MB. Osteoarthritis: a disease of the joint as an organ. Arthritis Rheum. 2012;64(6):1697-707.

4. Goldring SR. Alterations in periarticular bone and cross talk between subchondral bone and articular cartilage in osteoarthritis. Ther Adv Musculoskel Dis. 2012:4(4):249-58.

5. Belluzzi E, Stocco E, Pozzuoli A, Granzotto M, Porzionato A, Vettor R, et al. Contribution of infrapatellar fat pad and synovial membrane to knee osteoarthritis pain. Biomed Res Int. 2019;2019:6390182.

6. Zhang Y, Jordan JM. Epidemiology of osteoarthritis. Clin Geriatr Med. 2010;26(3):355-69.

7. Kobayashi T, Yoshihara Y, Yamada H, Fujikawa K. Procollagen IIC-peptide as a marker for assessing mechanical risk factors of knee osteoarthritis: effect of obesity and varus alignment. Ann Rheum Dis. 2000;59(12):982-4.

8. Hedbom E, Antonsson P, Hjerpe A, Aeschlimann D, Paulsson M, Rosa-

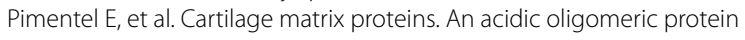
(COMP) detected only in cartilage. J Biol Chem. 1992;267(9):6132-6.

9. Saxne T, Heinegård D. Cartilage oligomeric matrix protein: a novel marker of cartilage turnover detectable in synovial fluid and blood. Br J Rheumatol. 1992;31(9):583-91.

10. Ju C, Liu R, Zhang Y, Zhang F, Sun J, Lv X-B, et al. Exosomes may be the potential new direction of research in osteoarthritis management. BioMed Res Intl. 2019;2019:7695768.

11. Zhang W, Nuki G, Moskowitz RW, Abramson S, Altman RD, Arden NK, et al OARSI recommendations for the management of hip and knee osteoarthritis: part III: changes in evidence following systematic cumulative update of research published through January 2009. Osteoarthr Cartil. 2010;18(4):476-99.

12. Hunter DJ, Nevitt M, Losina E, Kraus V. Biomarkers for osteoarthritis: current position and steps towards further validation. Best Pract Res Clin Rheumatol. 2014;28(1):61-71.

13. Kellgren JH, Lawrence JS. Radiological assessment of osteo-arthrosis. Ann Rheum Dis. 1957;16(4):494-502.

14. Swagerty DL Jr, Hellinger D. Radiographic assessment of osteoarthritis. Am Fam Physician. 2001;64(2):279-86.

15. Cahue S, Sharma L, Dunlop D, lonescu M, Song J, Lobanok T, et al. The ratio of type II collagen breakdown to synthesis and its relationship with the progression of knee osteoarthritis. Osteoarthr Cartil. 2007;15(7):819-23.

16. Watt FE. Osteoarthritis biomarkers: year in review. Osteoarthr Cartil. 2018;26(3):312-8

17. Mazières B, Garnero P, Guéguen A, Abbal M, Berdah L, Lequesne M, et al. Molecular markers of cartilage breakdown and synovitis at baseline as predictors of structural progression of hip osteoarthritis. The ECHODIAH Cohort. Ann Rheum Dis. 2006;65(3):354-9.

18. Bauer DC, Hunter DJ, Abramson SB, Attur M, Corr M, Felson D, et al. Classification of osteoarthritis biomarkers: a proposed approach. Osteoarthr Cartil. 2006;14(8):723-7.

19. Ishijima M, Watari T, Naito K, Kaneko H, Futami I, Yoshimura-Ishida K, et al. Relationships between biomarkers of cartilage, bone, synovial metabolism and knee pain provide insights into the origins of pain in early knee osteoarthritis. Arthritis Res Ther. 2011;13(1):R22. 
20. Verma P, Dalal K. Serum cartilage oligomeric matrix protein (COMP) in knee osteoarthritis: a novel diagnostic and prognostic biomarker. J Orthop Res. 2013;31(7):999-1006.

21. Heinegård D, Lorenzo P, Saxne T. Matrix glycoproteins and proteoglycans in cartilage. In: Harris ED, Budd RC, Firestein GS, Genovese MC, Ruddy S, editors. Kelley's textbook of rheumatology. Philadelphia: Elsevier Saunders; 2005.

22. Cibere J, Zhang H, Garnero P, Poole AR, LobanokT, Saxne T, et al. Association of biomarkers with pre-radiographically defined and radiographically defined knee osteoarthritis in a population-based study. Arthritis Rheum. 2009;60(5):1372-80.

23. Turan Y, Bal S, Gurgan A, Topac H, Koseoglu M. Serum hyaluronan levels in patients with knee osteoarthritis. Clin Rheumatol. 2007;26(8):1293-8.

24. Lotz M, Martel-Pelletier J, Christiansen C, Brandi M-L, Bruyère O, Chapurlat $R$, et al. Value of biomarkers in osteoarthritis: current status and perspectives. Ann Rheum Dis. 2013;72(11):1756-63.

25. Li Y, Yuan Z, Yang H, Zhong H, Peng W, Xie R. Recent advances in understanding the role of cartilage lubrication in osteoarthritis. Molecules. 2021;26(20):6122.

26. Tamer TM. Hyaluronan and synovial joint: function, distribution and healing. Interdiscip Toxicol. 2013;6(3):111-25.

27. Punzi L. The complexity of the mechanisms of action of hyaluronan in joint diseases. Clin Exp Rheumatol. 2001;19(3):242-6.

28. Dahl LB, Dahl IM, Engström-Laurent A, Granath K. Concentration and molecular weight of sodium hyaluronate in synovial fluid from patients with rheumatoid arthritis and other arthropathies. Ann Rheum Dis. 1985:44(12):817-22

29. Singh $S$, Kumar D, Sharma NR. Role of hyaluronic acid in early diagnosis of knee osteoarthritis. J Clin Diagn Res. 2014;8(12):LC04-7.

30. Davis CR, Karl J, Granell R, Kirwan JR, Fasham J, Johansen J, et al. Can biochemical markers serve as surrogates for imaging in knee osteoarthritis? Arthritis Rheum. 2007;56(12):4038-47.

31. Nelson F, Dahlberg L, Laverty S, Reiner A, Pidoux I, lonescu M, et al. Evidence for altered synthesis of type II collagen in patients with osteoarthritis. J Clin Invest. 1998:102(12):2115-25.

32. Kinoshita M, Kaneko H, Liu L, Sadatsuki R, Hada S, Shimura Y, et al. Association between the lower serum PIICP level and the joint space narrowing on the asymptomatic knee osteoarthritis in men in early frontiers. Osteoarthr Cartil. 2014;22:S367-8.

33. Sasaki E, Tsuda E, Yamamoto Y, Maeda S, Inoue R, Chiba D, et al. Serum hyaluronic acid concentration predicts the progression of joint space narrowing in normal knees and established knee osteoarthritis - a five-year prospective cohort study. Arthritis Res Ther. 2015;17(1):283.

34. Luyten FP, Bierma-Zeinstra S, Dell'Accio F, Kraus VB, Nakata K, Sekiya I, et al. Toward classification criteria for early osteoarthritis of the knee. Semin Arthritis Rheum. 2018:47(4):457-63.

35. Wang FF, Ha L, Yu HY, Mi L, Han JL, Gao W. Altered serum level of cartilage oligomeric matrix protein and its association with coronary calcification in patients with coronary heart disease. J Geriatr Cardiol. 2017;14(2):87-92.

36. Laurent TC, Laurent UBG, Fraser JRE. Serum Hyaluronan as a disease marker. Ann Med. 1996;28(3):241-53.

37. Adie S, Harris I, Chuan A, Lewis P, Naylor JM. Selecting and optimising patients for total knee arthroplasty. Med J Aust. 2019;210(3):135-41.

38. Altman R, Asch E, Bloch D, Bole G, Borenstein D, Brandt K, et al. Development of criteria for the classification and reporting of osteoarthritis. Classification of osteoarthritis of the knee. Diagnostic and therapeutic criteria Committee of the American Rheumatism Association. Arthritis Rheum. 1986;29(8):1039-49.

39. Rousseau JC, Zhu Y, Miossec P, Vignon E, Sandell LJ, Garnero P, et al. Serum levels of type IIA procollagen amino terminal propeptide (PIIANP) are decreased in patients with knee osteoarthritis and rheumatoid arthritis. Osteoarthr Cartil. 2004;12(6):440-7.

40. Leung YY, Huebner JL, Haaland B, Wong SBS, Kraus VB. Synovial fluid proinflammatory profile differs according to the characteristics of knee pain. Osteoarthr Cartil. 2017:25(9):1420-7.

41. Alghadir AH, Anwer S, Iqbal A, Iqbal ZA. Test-retest reliability, validity, and minimum detectable change of visual analog, numerical rating, and verbal rating scales for measurement of osteoarthritic knee pain. J Pain Res. 2018;11:851-6.
42. van Tunen JAC, Peat G, Bricca A, Larsen LB, Søndergaard J, Thilsing T, et al. Association of osteoarthritis risk factors with knee and hip pain in a population-based sample of 29-59 year olds in Denmark: a cross-sectional analysis. BMC Musculoskelet Disord. 2018;19(1):300.

43. Tuck MK, Chan DW, Chia D, Godwin AK, Grizzle WE, Krueger KE, et al. Standard operating procedures for serum and plasma collection: early detection research network consensus statement standard operating procedure integration working group. J Proteome Res. 2009;8(1):113-7.

44. Törmälehto S, Mononen ME, Aarnio E, Arokoski JPA, Korhonen RK, Martikainen J. Health-related quality of life in relation to symptomatic and radiographic definitions of knee osteoarthritis: data from osteoarthritis initiative (OAI) 4-year follow-up study. Health Qual Life Outcomes. 2018;16(1):154.

45. Sasaki E, Tsuda E, Yamamoto Y, Iwasaki K, Inoue R, Takahashi I, et al. Serum hyaluronan levels increase with the total number of osteoarthritic joints and are strongly associated with the presence of knee and finger osteoarthritis. Int Orthop. 2013;37(5):925-30.

46. Nemirovskiy OV, Sunyer T, Aggarwal P, Abrams M. Hellio Le Graverand MP, Mathews WR. Discovery and development of the $\mathrm{N}$-terminal procollagen type II (NPII) biomarker: a tool for measuring collagen type II synthesis. Osteoarthr Cartil. 2008;16(12):1494-500.

47. Stiebel M, Miller LE, Block JE. Post-traumatic knee osteoarthritis in the young patient: therapeutic dilemmas and emerging technologies. Open Access J Sports Med. 2014;5:73-9.

48. Messier SP. Obesity and osteoarthritis: disease genesis and nonpharmacologic weight management. Rheum Dis Clin N Am. 2008;34(3):713-29.

49. Berry PA, Maciewicz RA, Wluka AE, Downey-Jones MD, Forbes A, Hellawell CJ, et al. Relationship of serum markers of cartilage metabolism to imaging and clinical outcome measures of knee joint structure. Ann Rheum Dis. 2010;69(10):1816-22.

50. Garnero P, Ayral X, Rousseau JC, Christgau S, Sandell LJ, Dougados M, et al. Uncoupling of type II collagen synthesis and degradation predicts progression of joint damage in patients with knee osteoarthritis. Arthritis Rheum. 2002;46(10):2613-24.

51. Dahlberg L, Roos H, Saxne T, Heinegård D, Lark MW, Hoerrner LA, et al. Cartilage metabolism in the injured and uninjured knee of the same patient. Ann Rheum Dis. 1994:53(12):823-7.

52. Sharif M, Saxne T, Shepstone L, Kirwan JR, Elson CJ, Heinegård D, et al. Relationship between serum cartilage oligomeric matrix protein levels and disease progression in osteoarthritis of the knee joint. Br J Rheumatol. 1995;34(4):306-10.

53. Jiao Q, Wei L, Chen C, Li P, Wang X, Li Y, et al. Cartilage oligomeric matrix protein and hyaluronic acid are sensitive serum biomarkers for early cartilage lesions in the knee joint. Biomarkers. 2016;21(2):146-51.

54. Hunter DJ, Li J, LaValley M, Bauer DC, Nevitt M, DeGroot J, et al. Cartilage markers and their association with cartilage loss on magnetic resonance imaging in knee osteoarthritis: the Boston osteoarthritis knee study. Arthritis Res Ther. 2007;9(5):R108.

55. Dragomir AD, Kraus VB, Renner JB, Luta G, Clark A, Vilim V, et al. Serum cartilage oligomeric matrix protein and clinical signs and symptoms of potential pre-radiographic hip and knee pathology. Osteoarthr Cartil. 2002;10(9):687-91.

56. Sowers MF, Karvonen-Gutierrez CA, Yosef $M$, Jannausch $M$, Jiang $Y$, Garnero P, et al. Longitudinal changes of serum COMP and urinary CTX-II predict $X$-ray defined knee osteoarthritis severity and stiffness in women. Osteoarthr Cartil. 2009;17(12):1609-14.

57. Fernandes FA, Pucinelli ML, da Silva NP, Feldman D. Serum cartilage oligomeric matrix protein (COMP) levels in knee osteoarthritis in a Brazilian population: clinical and radiological correlation. Scand J Rheumatol. 2007:36(3):211-5

58. Goldring MB, Goldring SR. Articular cartilage and subchondral bone in the pathogenesis of osteoarthritis. Ann N Y Acad Sci. 2010;1192:230-7.

59. Mathiessen A, Conaghan PG. Synovitis in osteoarthritis: current understanding with therapeutic implications. Arthritis Res Ther. 2017:19(1):18.

60. Marini S, Fasciglione GF, Monteleone G, Maiotti M, Tarantino U, Coletta M. A correlation between knee cartilage degradation observed by arthroscopy and synovial proteinases activities. Clin Biochem. 2003:36(4):295-304

61. Elliott AL, Kraus VB, Luta G, Stabler T, Renner JB, Woodard J, et al. Serum hyaluronan levels and radiographic knee and hip osteoarthritis in African 
Americans and Caucasians in the Johnston County osteoarthritis project. Arthritis Rheum. 2005;52(1):105-11.

62. Kaneko H, Ishijima M, Doi T, Futami I, Liu L, Sadatsuki R, et al. Reference intervals of serum hyaluronic acid corresponding to the radiographic severity of knee osteoarthritis in women. BMC Musculoskelet Disord. 2013;14(1):34

63. Sharif M, George E, Shepstone L, Knudson W, Thonar EJ-MA, Cushnaghan $J$, et al. Serum hyaluronic acid level as a predictor of disease progression in osteoarthritis of the knee. Arthritis Rheum. 1995;38(6):760-7.

64. Inoue R, Ishibashi Y, Tsuda E, Yamamoto Y, Matsuzaka M, Takahashi I, et al. Knee osteoarthritis, knee joint pain and aging in relation to increasing serum hyaluronan level in the Japanese population. Osteoarthr Cartil. 2011;19(1):51-7.

65. Ding C, Cicuttini F, Scott F, Cooley H, Jones G. Association between age and knee structural change: a cross sectional MRI based study. Ann Rheum Dis. 2005;64(4):549-55.

66. Rostami S, Parsian H. Hyaluronic acid: from biochemical characteristics to its clinical translation in assessment of liver fibrosis. Hepat Mon. 2013;13(12):e13787.

67. Bihlet AR, Byrjalsen I, Bay-Jensen A-C, Andersen JR, Christiansen C, Riis BJ, et al. Associations between biomarkers of bone and cartilage turnover, gender, pain categories and radiographic severity in knee osteoarthritis. Arthritis Res Ther. 2019;21(1):203.

68. Karsdal MA, Byrjalsen I, Bay-Jensen AC, Henriksen K, Riis BJ, Christiansen C. Biochemical markers identify influences on bone and cartilage degradation in osteoarthritis--the effect of sex, Kellgren-Lawrence (KL) score, body mass index (BMI), oral salmon calcitonin (SCT) treatment and diurnal variation. BMC Musculoskelet Disord. 2010;11:125.

69. van Spil WE, Drossaers-Bakker KW, Lafeber FP. Associations of CTX-II with biochemical markers of bone turnover raise questions on its tissue origin: data from CHECK, a cohort study of early osteoarthritis. Ann Rheum Dis. 2013;72(1):29-36

70. Riddle DL, Stratford PW. Unilateral vs bilateral symptomatic knee osteoarthritis: associations between pain intensity and function. Rheumatology (Oxford). 2013;52(12):2229-37.

71. Arunrukthavon P, Heebthamai D, Benchasiriluck P, Chaluay S, Chotanaphuti T, Khuangsirikul S. Can urinary CTX-II be a biomarker for knee osteoarthritis? Arthroplasty. 2020;2(1):6.

72. Nees TA, Rosshirt N, Zhang JA, Reiner T, Sorbi R, Tripel E, et al. Synovial cytokines significantly correlate with osteoarthritis-related knee pain and disability: inflammatory mediators of potential clinical relevance. J Clin Med. 2019;8(9):1343.

\section{Publisher's Note}

Springer Nature remains neutral with regard to jurisdictional claims in published maps and institutional affiliations.

Ready to submit your research? Choose BMC and benefit from:

- fast, convenient online submission

- thorough peer review by experienced researchers in your field

- rapid publication on acceptance

- support for research data, including large and complex data types

- gold Open Access which fosters wider collaboration and increased citations

- maximum visibility for your research: over $100 \mathrm{M}$ website views per year

At BMC, research is always in progress.

Learn more biomedcentral.com/submissions 